\title{
Limited-Information Modeling of Loggerhead Turtle Population Size
}

\author{
John M. Grego \\ University of South Carolina \\ Department of Statistics
}

\author{
David B. Hitchcock \\ University of South Carolina \\ Department of Statistics
}

June 28, 2012

\begin{abstract}
We attempt to estimate the size of a population of female loggerhead turtles. In traditional capture-recapture experiments to estimate the size of an animal population, individual animals are tagged and the information about which individuals are captured repeatedly is crucial. For these loggerhead turtle data, information about individual turtles is not available. Rather, we observe only the counts of successful and failed nestings at a location over a series of days (in our case, three). We view the turtles' nesting behavior as an alternating renewal process, model it using parametric distributions, and then derive probability distributions that describe the behavior of the turtles during the three days via a 3-way contingency table. We adopt a Bayesian approach, formulating our model in terms of parameters about which strong prior information is available. We use a Gibbs sampling algorithm to sample from the posterior distribution of our random quantities, the most crucial of which is the number of turtles remaining offshore during the entire sampling period. We illustrate the method using a data set from loggerhead turtle sites along the South Carolina coast.
\end{abstract}


We provide a simulation study which illustrates the quality and robustness of the method and investigates sensitivity to prior parameter specification.

KEY WORDS: Bayesian methods, Capture-recapture, Ecological applications, Environmental applications, Gibbs sampling, Mark-recapture, Renewal process.

\section{Introduction}

The loggerhead sea turtle (scientific name: Caretta caretta) is listed as a threatened species in the United States under the Endangered Species Act (NOAA Office of Protected Resources 2011). In particular, loggerheads in the Atlantic Ocean from Florida to North Carolina have been reported as declining in number (Murphy and Griffin 2005). Thus having statistically sound estimates of loggerhead population size is a critical scientific goal. Reports of a decline in numbers are based in part on long-term surveys of nesting activity/nest abundance. Though these surveys were not originally designed to determine population size, we propose a method for estimating the population size of female loggerhead turtles using survey data designed instead to track nest abundance.

Traditional capture-recapture models have a long history of use in estimating the size of a population (e.g., an animal population) whose members cannot be counted exactly because, practically, many remain hidden to investigators. In such an experiment, a particular site is visited repeatedly (say, $T$ times), and individual animals that are captured at a site visit are tagged and released. Counts of captures for individual animals are made and are critical to inference about the population size. We describe a common classical approach to capture-recapture inference at the beginning of Section 3.

Otis et al. (1978) presented a comprehensive set of capture-recapture models ranging from a simple model assuming a unique probability of capture across visits and across animals to models allowing for various forms of variation in capture probability. 
This work was extended and generalized by Evans, Bonett, and McDonald (1994).

Capture-recapture research makes the distinction between closed populations (in which we assume no animals were born, died, or migrated into or out of the habitat during the study period) and open populations (which permit the possibility of deaths or new arrivals during the study period). Agresti (2002, Sec. 12.3.6 and Sec. 13.1.3) discusses closed-population capture-recapture models in the context of random-effects models and latent-class models. The random-effects presentation is based on a $2^{T}$ contingency table and is related to previous work by Fienberg (1972) and the log-linear modeling approach of Cormack (1989). Pledger et al. (2003) studied heterogeneity in capture and survival probabilities for an open population model using a finite mixture approach.

While our primary goal is to estimate an unknown population size, the datagathering mechanism we investigate in this paper is quite different than those in previous studies. Data gathered on loggerhead turtle abundance rarely involves tagging. The information we have is therefore far more limited than in a traditional capture-recapture situation. We consider adapting principles of capture-recapture analysis to the problem of estimating (in the absence of tagging) the population of female loggerhead turtles living off a given beach area.

The unique nesting pattern of these turtles plays a fundamental role in the analysis of the detection data. During their nesting season, loggerhead turtles come ashore at night when they are biologically ready to lay eggs, and only during these shore excursions can they be "counted." A turtle may nest successfully during the first night's attempt; on the other hand, the attempt may be unsuccessful, which is known as a "false crawl." False crawls may occur because of disturbances (e.g., from the presence of humans) or because of the turtle's momentary discomfort with an environmental factor such as the beach characteristics or temperature (Dodd 1988; Raymond 1984; Stoneburner and Richardson 1981). In some cases the reason for the false crawl is simply unknown (Dodd 1988). When a false crawl occurs, the turtle will return on 
successive nights, each night nesting or false-crawling. Once a nest is successfully made, the turtle returns to the ocean. Therefore, on each night, every turtle's status can be labeled by one of $\{$ Offshore, False Crawl, Nest $\}$ (abbreviated $\{\mathrm{O}, \mathrm{F}, \mathrm{N}\}$ ).

The data in such studies are typically gathered by researchers and volunteers who search the beach (on foot or via aerial surveys) early in the morning, looking for signs of the previous night's turtle activity. Evidence that indicates a turtle excursion includes crawl tracks in the sand leading from the ocean to the higher beach and the digging of a "body pit" and/or "egg chamber" in the sand (Dodd 1988). A successful nesting is further evidenced by eggs dropped in the chamber and the turtle's filling and concealing the body pit with sand before it returns to the ocean. A false crawl may consist of a brief trail through the sand, or it may include digging the body pit and/or egg chamber. Once the turtle begins to drop eggs, however, it usually completes the nesting process (Margaritoulis 1985).

Experienced researchers are typically good at distinguishing a false crawl from a nest, although these can appear similar to an untrained eye. The researchers count the number of successful nests and the number of false crawls from the previous night, typically gathering these counts on $D$ consecutive mornings during the nesting season. In this paper we assume that counts are available for at least $D=3$ consecutive nights during the middle of the season. In studies of nesting behavior, the ultimate goal is to estimate the total number of nests over the course of a season based on multiple sets of consecutive-day surveys. Using assumptions based on scientific knowledge of turtle nesting behavior, these surveys of nesting activity can actually be used to construct estimates of population size.

We assume turtles make their first onshore excursion of the season at somewhat evenly distributed times over the early weeks of the season (which will motivate a uniform distribution for the length of time before a nominal start of the season and the first arrival in our simulated data of Section 5). The mean number of excursions per season has been estimated to be around 3 or 4 by Frazer and Richardson (1985) 
and around 2.5 by Richardson and Richardson (1982). This number is not crucial for us, however, except to confirm the usual length of the season, since we are interested in turtle counts in the middle of the season. Turtles tend to remain in the same general area between nesting excursions (Limpus and Reed 1985); this justifies modeling the turtle population as closed, an assumption we will make henceforth.

Some key differences exist between the information available in the turtle study and that available in traditional capture-recapture studies. For example, the status for each animal in a traditional study is binary (Detected, Not Detected) whereas the turtles have three possible states (two of which represent a type of detection). Also, in traditional studies, detected animals are individually identified and tagged. The turtles are not seen individually, so we only have the total counts of the two "detection states" for each night.

\section{Model and Notation}

A key benefit of the method we will present is that our model is formulated in terms of parameters about which strong prior information (based on previous subject-matter studies and expert opinions) exists, i.e., the parameters that govern the turtles' biological nesting traits. Our approach permits us to take full advantage of this well-founded knowledge.

\subsection{Justification of Prior Distributions and Beliefs}

We make a few distributional assumptions about the data, which reasonably reflect expert opinion about the turtles' nesting behavior. We assume that the interarrival spacings (the numbers of days between a turtle's nesting and the next time she comes ashore to nest again) follow a normal distribution (technically an integer normal). While the mean $\mu$ and standard deviation $\sigma$ of this distribution are unknown, biol-

ogists strongly believe $\mu$ to be near 13 days (Dodd 1988; Talbert et al. 1980). We 
therefore choose a $N\left(\mu_{\mu}, \sigma_{\mu}^{2}\right)$ prior distribution for $\mu$, with $\mu_{\mu}=13$ and $\sigma_{\mu}^{2}=0.75^{2}$. Gallagher et al. (1972) suggest 12-14 days as the typical set of values this interarrival time takes; using the rule of thumb (McClave and Sincich 2006) of approximating the standard deviation as Range/4, we may assume $\sigma$ is near 0.75 . We place an inverse gamma (IG) prior on $\sigma$ with parameters $\alpha_{\sigma}=9$ and $\beta_{\sigma}=6$ for a prior expected value $E(\sigma)=6 /(9-1)=0.75$.

We also assume the number of false crawls before a successful nesting is modeled as a geometric random variable, with nesting probability $p$ and false crawl probability $q=1-p$. The geometric model assumes that the probability of false crawling is constant across nights, which are assumed independent. This independence assumption appears justified (Griffin, Personal communication, 2009), at least for several consecutive nights. Note that the probability of a large series of false crawl nights in a row (after which point $p$ might change) is fairly negligible. (The issue of whether $p$ is constant or random across turtles is addressed in Section 5.2.) We may obtain a prior expectation for $p$ based on scientific literature. Dodd (1980) gives estimates from a variety of sources of the proportion of onshore excursions that are false crawls. Talbert et al. (1980) gave such a value as 40.5\%, using South Carolina coastal data. Stoneburner (1981) gave figures of $30.8 \%, 41.2 \%$, and $50.4 \%$ for three locations along the southeastern Atlantic coast. Raymond (1984) and Crouse (1984) report roughly half of excursions resulted in false crawls. All in all, this prior information indicates the proportion of excursions that are false crawls is near 0.4. Since the geometric mean, $q / p$, represents the expected number of false crawls before the first nesting, a method-of-moments approach would equate 0.4 to $(q / p) /(q / p+1)$, where the denominator is the expected number of total excursions (false crawls plus nesting). Solving for $p$ simply results in an estimated prior expectation of $p=0.6$, i.e, the overall proportion of excursions judged to be nests serves as a logical prior guess of this constant nesting probability. (This can also be justified by the memoryless property of the geometric distribution.) Our prior on $p$ is a beta $(6,4)$, which implies $E(p)=0.6$ 
and $P[0.35<p<0.85] \approx 0.9$ a priori.

In Section 4, we will develop a prior distribution for the number of turtles offshore all three sampled nights.

\subsection{Marginal, Conditional, and Joint Probabilities}

Since the "nesting state" lasts one night, we can write marginal probabilities of each of the three states $\mathrm{O}, \mathrm{F}, \mathrm{N}$. These expressions, which represent the proportion of time a turtle can be expected to be in each state, are justified by treating the process as a 3-state alternating renewal process (see Ross 1996). These proportions are the expected lengths of time in each state, divided by the sums of the three expected lengths. Note that the expected time offshore is the normal mean $\mu$; the expected number of nights of false crawls is the geometric mean $q / p$; and the expected number of nights nesting is the constant 1 :

$$
\begin{aligned}
& p_{O}=\frac{\mu}{\mu+1+q / p} \\
& p_{N}=\frac{1}{\mu+1+q / p} \\
& p_{F}=\frac{q / p}{\mu+1+q / p}
\end{aligned}
$$

While the above representation of the marginal probabilities of each state technically holds for nonlattice random variables, it should be approximately correct for lattice random variables such as the "number of days." We point out that this model works best for data gathered within the interior of the nesting season: The initial time to nesting may follow a different distribution than do the interarrival times after a turtle has nested and has eased into its "equilibrium pattern" for that season; i.e., the process for the entire season is actually a delayed renewal process (Ross 1996). We note that the counts of turtle activity are typically gathered during the middle of the nesting season.

In addition, specific biological properties of the nesting process yield conditional 
probabilities for the states, given below (where $p_{B \mid A}$ denotes the probability of being in state $B$ on day $j$ given the turtle was in state $A$ on day $j-1$ ):

$$
\begin{array}{lll}
p_{O \mid O}=\omega & p_{N \mid O}=p(1-\omega) & p_{F \mid O}=q(1-\omega) \\
p_{O \mid N}=1 & p_{N \mid N}=0 & p_{F \mid N}=0 \\
p_{O \mid F}=0 & p_{N \mid F}=p & p_{F \mid F}=q
\end{array}
$$

The parameter $\omega$, the probability that an offshore turtle remains offshore, plays an important role in our model. Given a pair $(\mu, \sigma)$, we can calculate $\omega$, as we show in Section 2.3, and thus our solid prior knowledge of $(\mu, \sigma)$ yields a trustworthy prior expectation for $\omega$.

Using these marginal and conditional probabilities, we can construct joint probabilities for the possible successive three-day outcomes $A B C$, where $A \in\{O, F, N\}, B \in$ $\{O, F, N\}, C \in\{O, F, N\}$ (see Table 1). In deriving these joint probabilities we assume a kind of Markov property, assuming that $p_{C|B| A}=p_{C \mid B}$, so that $p_{A B C}=$ $p_{A} p_{B \mid A} p_{C \mid B}$. (We assume one practical exception to this property, setting $p_{O|O| N}=1$ rather than $p_{O|O| N}=p_{O \mid O}=\omega$, since a turtle who nested on the first observed day will virtually certainly remain offshore on the following two days.)

[[Table 1 goes around here]]

\subsection{Calculation of Probability of Remaining Offshore}

For given $\mu$ and $\sigma$, calculating $\omega=p_{O \mid O}$ is possible. This expression depends on the number of days since the last nesting episode (denoted $k$ in the following equation), so it may be expressed as a weighted average of discrete normal hazards:

$$
\omega \approx 1-\sum_{k=1}^{\infty} \frac{\Phi\left(\frac{k+0.5-\mu}{\sigma}\right)-\Phi\left(\frac{k-0.5-\mu}{\sigma}\right)}{1-\Phi\left(\frac{k+0.5-\mu}{\sigma}\right)} g\left(\frac{k-0.5-\mu}{\sigma}\right),
$$

where the 0.5 values are continuity corrections, since the data are recorded in days (a discrete variable). The density $g(\cdot)$ is a "waiting density." Note that for any given 
offshore turtle, we do not know how long it has been since the last nesting episode. The mean waiting time (say $U$ ) until the next onshore excursion could vary randomly from 0 days (if the turtle last nested long ago and is ready to nest again) to $\mu$ days (if the turtle just finished nesting). Specifically, if

$$
U \sim U(0, \mu), X \mid U \sim \max \left\{0, N\left(U, \sigma^{2}\right)\right\}
$$

then $g(\cdot)$ results from integrating $U$ out of the joint density of $(X, U)$.

Given our prior expectations that $\mu=13, \sigma=0.75$, we evaluate (1) - in practice truncating the infinite sum at a reasonable cutoff - and obtain the prior expected value for $\omega \approx 0.923$ in our case. This implies a prior expectation that just over $90 \%$ of turtles offshore one night will remain offshore the following night. Plugging prior expectations for $\mu, p$, and $\omega$ into the expression in the top left cell of Table 1 yields the prior expected $p_{O O O}=0.755$. Previous years' data (or expert guesses) about total population size can be combined with this value to develop a prior for the number of turtles offshore all three sampled nights, as we will do in Section 4.

\section{Estimation Method}

Traditional capture-recapture analysis observes animals over a period of $D$ days, recording counts $n_{s_{1} s_{2} \cdots s_{D}}$, where $s_{j}$ is the status (Present or Absent) for each day. For example, let $0=$ Absent and $1=$ Present. Then (in a three-day study, say) $n_{010}$ would be the counts of animals that were absent on Days 1 and 3 and spotted on Day 2. All such counts would be observed except $n_{000}$, the count of animals that were unseen on each day of the study. One traditional approach uses a Rasch-type (1961) model

$$
\operatorname{logit}\left[P\left(Y_{i j}=1 \mid u_{i}\right)\right]=u_{i}+\beta_{j}
$$

to model the log-odds of capture for animal $i$ on day $j$, where $u_{i} \sim N\left(0, \sigma^{2}\right)$ is an animal-specific random effect, and $\beta_{j}$ represents capturability on day $j$ (Agresti 
2002). A marginal likelihood can be constructed from the probability density function of $\mathbf{Y}=\left\{Y_{i j}\right\}$, by integrating over the random effects distribution. Model parameters are estimated using marginal maximum likelihood exclusively for the observed counts. Either conditional maximum likelihood or profile maximum likelihood approaches can then be used to estimate the unobserved cell count $n_{000}$. This estimator is added to the observed cell counts to obtain an estimate of the total population size $N_{t o t}$. For an overview of standard capture-recapture analyses under a variety of model assumptions, see Otis et al. (1978).

Recall that our situation differs from classical capture-recapture, in part, because we do not have the observed counts in the 3-way data table that corresponds to the table of joint 3-day cell probabilities. Rather, we observe the 6 -element vector $\mathbf{y}=\left(N_{1}, N_{2}, N_{3}, F_{1}, F_{2}, F_{3}\right)^{\prime}$, where $N_{i}, i=1,2,3$ is the total count of Nestings for day $i$, and $F_{i}, i=1,2,3$ is the total count of False Crawls for day $i$. One approach is to treat this observed vector $\mathbf{y}$ as a set of fixed marginals, and apply the profile likelihood approach for the Rasch-type model to the interior cells of the 3-way table to estimate a total count by using the relationships among the cell probabilities in Table 1 to estimate the unknown parameters $(p, \mu, \omega)$ in Table 1 . Such classical likelihood approaches produce stable estimates of the parameter $p$, the parameter product $\mu(1-\omega)$ and the interior cell counts in Table 1 . However, estimates of parameters $\mu$ and $\omega$ and hence $N_{\text {tot }}$ are not stable, primarily due to identifiability issues involving $\mu$ and $\omega$. Intuitively, the product $\mu(1-\omega)$ represents the rate at which turtles arrive onshore, and the rate, which can remain constant as $\mu$ and $\omega$ are manipulated, is the only information about $\mu$ and $1-\omega$ we can retrieve from the marginal cell counts.

Furthermore, $\mathbf{y}$ is not a genuine multinomial observation since a given turtle could contribute to more than one cell of $\mathbf{y}$. For example, a turtle could false crawl on days 1 and 2 and nest on day 3 , contributing to each of the $F_{1}, F_{2}$, and $N_{3}$ counts. 


\subsection{Development of Model for Number of Days Onshore}

Given the problems with the classical likelihood approach, the natural limitations inherent in our observed data, and the strong prior information available about the turtles' biological processes, we adopt a Bayesian analysis. Bayesian methods for traditional capture-recapture analyses have been proposed (Gazey and Staley 1986; George and Robert 1992; Dupuis 1995; Brooks et al. 2000); see also Cummings (2011) for related references. The nontraditional structure of the turtle data necessitates a somewhat different approach than those.

Recall $\mathbf{y}=\left(N_{1}, N_{2}, N_{3}, F_{1}, F_{2}, F_{3}\right)^{\prime}$ is the observed data vector. Let

$$
\mathbf{p}=\left(p_{O O O}, p_{O O F}, p_{O O N}, p_{O F F}, p_{O F N}, p_{O N O}, p_{F F F}, p_{F F N}, p_{F N O}, p_{N O O}\right)^{\prime}
$$

be the vector for the cell probabilities in Table 1, i.e., for a 10-category (unobserved) multinomial random variable. Then define $C \in\{0,1,2,3\}$ to be the number of cells in $\mathbf{y}$ to which a particular turtle contributes. That is, $C_{i}=$ the number of days onshore (out of three) for turtle $i$. Each cell in Table 1 can be assigned to one value of $C$, yielding the unconditional distribution of $C$, which is multinomial with probabilities:

$$
\begin{aligned}
& P(C=0)=p_{O O O} \\
& P(C=1)=p_{N O O}+p_{O N O}+p_{O O N}+p_{O O F} \\
& P(C=2)=p_{F N O}+p_{O F N}+p_{O F F} \\
& P(C=3)=p_{F F N}+p_{F F F}
\end{aligned}
$$

Define the vector $\mathbf{m}=\left(m_{1}, m_{2}, m_{3}\right)^{\prime}$ to contain counts of the number of unique turtles for which $C=i$, for $i=1,2,3$. (Note $m_{0}$, the number of turtles for which $C=0$, is simply $N_{O O O}$.) The individual counts in $\mathbf{m}$ are unknown; however, we know the total number of unique observed turtles is $N_{o b s}=\sum_{i=1}^{3} m_{i}=N_{1}+N_{2}+N_{3}+F_{3}$, which is determined from the observed $\mathbf{y}$. 


\subsection{Gibbs Sampling Approach}

Using a Bayesian approach, we consider the collection of unknown quantities in our problem to be $\left(\mathbf{m}, N_{O O O}, \mu, \sigma, p\right)$. We will use a Gibbs sampling approach to approximate the joint posterior $f\left(\mathbf{m}, N_{O O O}, \mu, \sigma, p \mid \mathbf{y}\right)$ by sampling iteratively from the respective full conditional distributions. The full conditionals we use here are strongly motivated by specific prior knowledge of turtle behavior.

Given $N_{O O O}, \mu, \sigma, p, \mathbf{y}$, determining $\mathbf{m}$ is tantamount to allocating the $N_{o b s}$ turtles into three classes with probabilities proportional to $(P(C=1), P(C=2), P(C=3))$. So the full conditional of $\mathbf{m}$ is a three-category multinomial:

$$
f\left(\mathbf{m} \mid N_{O o O}, \mu, \sigma, p, \mathbf{y}\right) \sim M N\left(N_{o b s} ; P(C=1) / K, P(C=2) / K, P(C=3) / K\right),
$$

where $K=\sum_{i=1}^{3} P(C=i)$.

By Bayes' theorem, $f\left(N_{O O O} \mid \mathbf{m}, \mu, \sigma, p, \mathbf{y}\right) \propto L\left(N_{O o O} \mid \mathbf{m}, \mu, \sigma, p, \mathbf{y}\right) f\left(N_{O o O}\right)$ where $L(\cdot \mid \cdot)$ denotes a likelihood and $f\left(N_{O O O}\right)$ is the prior for $N_{O O O}$. Considering turtles to be "successes" or "failures" according to whether they came onshore at all or not, respectively, we treat $N_{O O O}$ as a count of "failures" at the point of $N_{o b s}=$ $\sum_{i=1}^{3} m_{i}$ successes. Thus we model $N_{O O O} \mid \mathbf{m}, \mu, \sigma, p, \mathbf{y}$ as negative binomial with "size" parameter $r=N_{o b s}$ and success probability $1-p_{O O O}$ (note $p_{O o O}$ is a function of $\mu$, $\sigma$, and $p$ ). To incorporate prior knowledge about $N_{O O O}$, we use a negative binomial; the prior parameters for our specific data set are justified in Section 4. So the full conditional of $N_{O O O}$ is

$$
f\left(N_{O O O} \mid \mathbf{m}, \mu, \sigma, p, \mathbf{y}\right) \propto N B\left(N_{O O O}, N_{o b s}, 1-p_{O O O}\right) \times N B\left(N_{O O O}, r_{\mathrm{pri}}, p_{\mathrm{pri}}\right),
$$

where $N B\left(\cdot, r\right.$, prob) denotes a negative binomial density and $r_{\text {pri }}$ and $p_{\text {pri }}$ are prior parameters. This full conditional is not a standard distribution, but one can sample from it via an Accept-Reject algorithm.

Our interarrival mean $\mu$ is assumed independent of $\sigma$, so that $f\left(\mu \mid N_{O O O}, \mathbf{m}, p, \sigma, \mathbf{y}\right)=$ $f\left(\mu \mid N_{O O O}, \mathbf{m}, p, \mathbf{y}\right)$. Our expression for the full conditional for $\mu$ is motivated by the 
fact that — as argued in the Appendix — the information about $\mu$ in $\left(N_{O o O}, \mathbf{m}, \mathbf{y}\right)$ is contained in the relative sizes of $N_{o b s}$ and $N_{O O O}$, or specifically in the ratio $N_{\text {obs }} /\left(N_{O O O}+N_{o b s}\right)$, so that:

$$
\begin{aligned}
f\left(\mu \mid N_{O O O}, \mathbf{m}, p, \mathbf{y}\right) & \propto f\left(N_{O O O}, \mathbf{m}, p, \mathbf{y} \mid \mu\right) f(\mu) \\
& \propto f\left(N_{o b s} /\left(N_{O O O}+N_{o b s}\right) \mid p, \mu\right) f(p \mid \mu) f(\mu) \\
& \propto f\left(N_{o b s} /\left(N_{O O O}+N_{o b s}\right) \mid p, \mu\right) f(\mu)
\end{aligned}
$$

since the distribution $f(p \mid \mu)$ is actually free of $\mu$. The details of the derivation of the full conditional are given in the Appendix. In summary, the full conditional for $\mu$ is normal and incorporates information from the normal prior on $\mu$ and from the data and $N_{O O O}$ (via the ratio $N_{o b s} /\left(N_{O O O}+N_{o b s}\right)$ ):

$$
f\left(\mu \mid N_{O O O}, \mathbf{m}, \sigma, p, \mathbf{y}\right) \sim N\left(\frac{\frac{\mu_{\mu}}{\sigma_{\mu}^{2}}+\frac{M}{V}}{\frac{1}{\sigma_{\mu}^{2}}+\frac{1}{V}}, \frac{\sigma_{\mu}^{2} V}{\sigma_{\mu}^{2}+V}\right)
$$

where $M=(3+q / p)\left(N_{O o O}+N_{o b s}\right) / N_{o b s}-(1+q / p)$ and $V=\left[(3+q / p)^{2}\left(N_{O o O}+\right.\right.$ $\left.\left.N_{o b s}\right) N_{O O O}\right] / N_{o b s}^{3}$.

We assume $\sigma$ is independent of $\mu$ and $p$ so that the full conditional for $\sigma$ is

$$
f\left(\sigma \mid N_{O O O}, \mathbf{m}, \mu, p, \mathbf{y}\right) \propto f\left(N_{O O O}, \mathbf{m}, \mathbf{y} \mid \sigma\right) f(\sigma)
$$

Similarly as argued for $\mu$ above, the information about $\sigma$ in $\left(N_{O O O}, \mathbf{m}, \mathbf{y}\right)$ is in the proportion $N_{O O O} /\left(N_{O O O}+N_{o b s}\right)$. Modeling that proportion with a normal distribution and choosing $f(\sigma)$ to be inverse gamma, the full conditional for $\sigma$ is:

$$
f\left(\sigma \mid N_{O O O}, \mathbf{m}, \mu, p, \mathbf{y}\right) \propto Z(z(\sigma)) \times I G\left(\sigma, \alpha_{\sigma}, \beta_{\sigma}\right),
$$

where $Z(\cdot)$ denotes a standard normal density, $I G(\cdot, \alpha, \beta)$ denotes an inverse gamma density and

$$
z(\sigma)=\frac{N_{O O O} /\left(N_{O O O}+N_{o b s}\right)-p_{O O O}}{\sqrt{p_{O O O}\left(1-p_{O O O}\right) /\left(N_{O O O}+N_{o b s}\right)}}
$$


is a standardized version of the proportion of turtles that came onshore. (Recall that $p_{O o O}$ depends on $\sigma$ through $\omega$.) This full conditional is not a standard distribution, but again one can sample from it via Accept-Reject.

The nesting probability $p$ is assumed independent of $\sigma$, so we have

$$
\begin{aligned}
f\left(p \mid N_{O O O}, \mathbf{m}, \mu, \sigma, \mathbf{y}\right) & =f\left(p \mid N_{O O O}, \mathbf{m}, \mu, \mathbf{y}\right) \\
& \propto f\left(N_{O O O}, \mathbf{m}, \mu, \mathbf{y} \mid p\right) f(p) \\
& =f\left(N_{O O O}, \mathbf{m}, \mu \mid p\right) f\left(\mathbf{y} \mid N_{O O O}, \mathbf{m}, \mu, p\right) f(p)
\end{aligned}
$$

The information in $\left(N_{O O O}, \mathbf{m}\right)$ about $p$ is contained in the ratio $N_{o b s} /\left(N_{O O O}+N_{o b s}\right)$, so the first piece of (3) is the same as the full conditional for $\mu$, but with $p$ as the random variable rather than $\mu$. Based on the observed vector $\mathbf{y}$, the totals of nests and false crawls for the three days, $N_{1}+N_{2}+N_{3}$ and $F_{1}+F_{2}+F_{3}$ are sufficient statistics for the nesting probability $p$. Treating excursions as Bernoulli trials (independent by our geometric model assumption), we model the nest count $N_{1}+N_{2}+N_{3}$ as binomial with success probability $p$. So the second piece of (3) is proportional to

$$
p^{N_{1}+N_{2}+N_{3}}(1-p)^{F_{1}+F_{2}+F_{3}}
$$

Augmented with the beta prior for $p$, we see the full conditional for $p$ is proportional to the density from $(2)$ (treated as a function of $p)$ times a beta $\left(N_{1}+N_{2}+N_{3}+\alpha_{p}, F_{1}+\right.$ $\left.F_{2}+F_{3}+\beta_{p}\right)$ density. We can again sample from this using Accept-Reject.

Given these full conditional expressions, we provide initial values for $\left(N_{O O O}, \mu, \sigma, p\right)$ and cycle through, sampling from each full conditional in turn. The main subchain of interest is the one for $N_{O o O}$, since our parameter of interest is $N_{t o t}=N_{O O O}+N_{o b s}$ (recall $N_{o b s}$ is known). Thus from the sampled $N_{O O O}$ values, we can make inference about $N_{\text {tot }}$. We now show how our method can be applied to a real data set. 


\section{Application to Real Three-Day Nesting Data}

The loggerhead turtle nesting season runs from roughly May to August (Dodd 1988). While turtle excursions may be haphazard at the beginning of the season, during the middle of the season, the patterns will have progressed into a kind of equilibrium state that should be well approximated by our model. Table 5 lists a typical observed data set. It gives raw counts of false crawls and nests observed over three consecutive days by South Carolina Department of Natural Resources researchers for June 16-18, 2000 on a particular set of South Carolina beaches.

Recall that our priors for $\mu, \sigma$, and $p$ were given in Section 2.1. Note that our prior expectations for $\mu, \sigma$, and $p$ imply a prior expected $p_{O O O}=0.755$. The South Carolina Department of Natural Resources (SCDNR) provides — based on standardized ground surveys - seasonal counts (starting in 1982) of nests on selected "index beaches" (http://www.dnr.sc.gov/seaturtle/ibs.htm). These index beaches are only a subset of the set of all South Carolina beaches that we are considering; SCDNR estimates that the index count is roughly $41 \%$ of nesting activity. For the five years previous to 2000, an annual average of around 1400 nests were counted on the index beaches. Thus a prior expected number of nests on the larger set of beaches is $1400 / 0.41 \approx 3415$. Turtles nest multiple times per season; expert guesses of the average number of nests per female range from 2.5 to around 4 (Frazer and Richardson 1985; Richardson and Richardson 1982). A best prior guess for the total number of females in the nesting area could be, say, 3415/3.25 $\approx 1051$. A reasonable interval for this guess would be $(3415 / 4,3415 / 2.5) \approx(854,1366)$. Thus we set the prior expectation for $N_{O O O}$ be $0.755 \times 1051 \approx 794$ and assume a priori that $N_{O O O}$ is likely within $(0.755 \times 854,0.755 \times 1366) \approx(645,1031)$. We choose a negative binomial prior for $N_{O O O}$ having mean 794 and size $r=80$, which satisfies our conditions nicely.

[[Table 5 goes around here]]

The observed number of unique turtles $N_{o b s}$ for this sample was $N_{1}+N_{2}+N_{3}+$ 
$F_{3}=77+76+71+72=296$, so 296 was added to each value in the subchain of $N_{O O O}$ values to create a sample of $N_{\text {tot }}$ values. We ran the Gibbs sampler for 10500 iterations and removed the first 500 values as burn-in. To reduce some very modest autocorrelation, we then thinned the sample by taking every second value of $N_{t o t}$. The resulting autocorrelation plot, shown in Figure 1 (bottom), indicated no serious autocorrelation among sampled values. A time series plot is given in Figure 1 (top), showing apparently fast convergence and mixing for the chain.

[[Figure 1 goes around here]]

A histogram of the remaining $5000 N_{\text {tot }}$ values is shown in Figure 2. The posterior sample is unimodal, mound-shaped, and symmetric. Our point estimate of $N_{t o t}$ is the median of the $N_{\text {tot }}$ values, which is 1123 in this case. A $90 \%$ credible interval for $N_{\text {tot }}$ is $(1031,1227)$, constructed from the 0.05 and 0.95 quantiles of the $N_{\text {tot }}$ values.

[[Figure 2 goes around here]]

As an informal judgment about whether our estimate is reasonable, we note from the literature two estimates of nesting activity during the 2000 season. Murphy and Griffin (2005) give a rough estimate (based on aerial surveys) of around 3400 total nests throughout the 2000 season. In addition, the South Carolina Department of Natural Resources provides — based on standardized ground surveys a count for the 2000 season of around 1150 nests for a group of selected "index beaches" (http://www.dnr.sc.gov/seaturtle/ibs.htm). Our three-day data includes counts from these index beaches, plus numerous other South Carolina beaches. In our data set, $36.2 \%$ of the nests (81 out of $N_{1}+N_{2}+N_{3}=224$ total nests) came from those index beaches. Dividing the seasonal index-beach nest count of 1150 by 0.362 gives a rough estimate of around 3180 total nests on our (larger) set of beaches.

With an approximate total nest count of either 3400 or 3180, our interval estimate of between 1031 and 1227 female turtles implies roughly 2.5 to 3.5 nesting episodes per season per turtle, on average, which lines up well with previous estimates (Frazer and Richardson 1985; Richardson and Richardson 1982). In short, our estimate of 
the population size is, at the very least, reasonable.

\section{Simulation Study}

In this section we describe a simulation study designed to gauge our method's performance on simulated data sets following our model (and data having some departures from our model). The twin purposes of these simulations are to investigate (1) the sensitivity of our procedure to prior specifications and (2) the robustness of the method to a violation of our geometric model assumption. We simulated full-season data records for a population of $N_{\text {tot }}=1000$ turtles in the following way. For the $i$ th turtle, $\left(i=1, \ldots, N_{t o t}\right)$, we generated a sequence of states, where each state value was one of $\{O, F, N\}$ (representing Offshore, False Crawl, Nest). For any particular turtle, the sequence was obtained by

1. Generate a string of $O$ 's of length $[U\rceil$, where $U \sim U(0,30)$. (This implies a turtle's first onshore arrival of the season occurred randomly between day 1 and day 30.)

2. Follow this with a string of $F$ 's whose length follows a geometric $(p)$ distribution.

3. Follow this with a single $N$.

4. Follow this with a string of $O$ 's of length $\lceil W\rceil$, where $W \sim N\left(\mu, \sigma^{2}\right)$.

5. Repeat steps (2)-(4) until 100 "days" have been generated for each turtle.

For these simulated data, we set the true values of our parameters to be $\mu=13$, $\sigma=0.75$, and $p=0.6$. Once the entire season of records was generated, a sequence of three consecutive days (the starting day was selected at random from day 50 through day 98 so that the selected days would be well into the "season") was chosen. (We are only interested in generating data that match reality for the middle of the season, not for the end of the season when turtle activity would tail off.) The counts of $F$ 's 
and N's were taken across all the turtle records for each of the three days, resulting in a simulated data set whose structure resembled that of Table 5.

Each of 200 such simulated data sets was analyzed using the approach outlined in Section 3. For each data set, a point estimate (posterior median) for $N_{t o t}$ and a $90 \%$ credible interval for $N_{\text {tot }}$ were obtained based on 10000 Gibbs iterations (10500 iterations were done overall, with the first 500 discarded as burn-in). To reduce modest autocorrelation (noted in our real data analysis) we thinned the sample by taking every other value of $N_{t o t}$.

Knowing the true population size (1000), we calculated (1) the empirical coverage probability; (2) the average (across the 200 simulated sets) width of the interval; and (3) the average bias of the posterior median.

To examine the effect of various factors on the method's performance, the simulations were run for a variety of settings:

- We varied the values of the prior mean for $\mu$, allowing prior means for $\mu$ that were alternately lower and higher than the true value of $\mu$. Simultaneously we varied the prior variance for $\mu$, allowing the prior for $\mu$ to be alternately more or less informative.

- We varied the values of the prior mean for $N_{O O O}$, allowing prior means for $N_{O O O}$ that were alternately lower and higher than the true value. Simultaneously we varied the size parameter for the prior on $N_{O O O}$, allowing the prior for $N_{O O O}$ to be alternately more or less informative.

- We generated data in which the nesting probability $p$ was different for each turtle. Rather than fixing $p=0.6$ in the population, we generated distinct $p_{i}$ values from (various) beta distributions having mean $0.6, i=1, \ldots, N_{\text {tot }}$.

\subsection{Gauging the Sensitivity to the Priors for $\mu$ and $N_{O O O}$}

[[Table 2 goes around here]] 
When the prior expected value for $\mu$ matches 13 , the true $\mu$ of the simulated data, the empirical coverage probability is very good, slightly higher than the nominal level of 0.90 . The point estimator appears essentially unbiased in this case. The average width of the credible intervals depends primarily on the value of $\sigma_{\mu}$, which measures the spread of the prior distribution. Naturally, a more informative prior (having lower $\sigma_{\mu}$ ) yields a narrower credible interval on average, while a more diffuse prior (having higher $\sigma_{\mu}$ ) yields wider credible intervals. When the prior is "mis-centered" so that $\mu_{\mu} \neq 13$, the coverage probability suffers slightly and the posterior median reveals a bias in the direction of the (misspecified) prior expected value. These problems are mitigated when the prior is made more diffuse (e.g., the bottom row of Table 2), in which case the method is remarkably robust to a misspecified $\mu_{\mu}$. It is quite natural that a tighter prior leads to prior information dominating and a more diffuse prior places more weight on data information. We recommend that an analyst having little confidence in the choice of prior expectation $\mu_{\mu}$ simply choose a fairly large $\sigma_{\mu}$ to decrease the effect of the prior. For our turtle data in Section 4 , we believe $\mu_{\mu}=13$ and $\sigma_{\mu}=0.75$ are justified.

[[Table 3 goes around here]]

When the prior expected value for $N_{O O O}$ is correctly specified at $755(=0.755 \times$ 1000), the empirical coverage probability is slightly higher than the nominal level. Even when the prior mean for $N_{O O O}$ is misspecified, the coverage probability remains high, especially when the prior is chosen to be fairly diffuse (note that a smaller "size" parameter implies a more diffuse prior distribution). Point estimation may be somewhat biased with a misspecified prior mean. Again, if the researcher is uncertain about the prior mean of $N_{O O O}$, a wide prior (having small "size") should be chosen; this yields wider credible intervals with at least nominal coverage. 


\subsection{Gauging the Effect of Allowing $p$ to Vary Across Turtles}

Fixing the prior on $\mu$ as $N\left(13,0.75^{2}\right)$ and the prior on $N_{O O O}$ to have mean 755 and size 80 , and generating data such that $\mu=13$ and $\sigma=0.75$, we allowed $p$ to vary across turtles, $p_{i} \sim \operatorname{beta}(a, b), i=1, \ldots, N_{t o t}$, such that $E\left(p_{i}\right)=0.6$. The results are given in Table 4.

[[Table 4 goes around here]]

Our estimation method is based on a constant $p$. When $p$ varies across turtles, the results are similar to the case when $p$ is constant. Thus if in reality the nesting probability varies across turtles, using our method should not be detrimental.

\section{Discussion}

The length $D$ of the series of days for which counts are available merits further discussion. We have assumed that counts of nestings and false crawls are available for at least three consecutive days. We could have obtained (or chosen to use) only two consecutive days of counts; we could then construct a $3 \times 3$ two-way table of joint probabilities similar to Table 1. The counts in the two-day table, however, do not incorporate as much information on false crawl behavior as the counts in the threeday table. Specifically, if the probability of a nesting is $p \approx 0.6$, then the additional information on $p$ gained from a third day of observation is substantive under the assumption of a geometric distribution for false crawl/nesting behavior.

On the other hand, counts could conceivably be gathered for $D \geq 4$ consecutive days. We did not explore this scenario in this article for reasons both practical and mathematical. First of all, the turtle survey is limited by logistical considerations. The consecutive-day survey takes place only during spring tides, which occur during the full moon and new moon (Hopkins-Murphy et al. 2001). Spring tides produce extreme high and low tides, and researchers favor days when the extreme low tides occur in the morning, thus exposing more of the beach, and hence providing better 
conditions for detecting turtle activity. This specific set of conditions lasts for only a few days.

Secondly, the crucial table of joint probabilities would have $3^{D}$ cells -81 for a four-day study, for example. While many of these cells would be structural zeroes, clearly the analysis becomes somewhat more unwieldy for four or more days. And we can appeal to the behavior of a geometric random variable once again to note that little additional information about false crawl behavior would be gained by including a fourth day in the study for the values of $p$ assumed in this study.

In the most recent decade or two, statisticians have often sought effective methods of analysis (e.g., data mining) of massive data sets. The problem presented here is quite the opposite scenario: We want to demonstrate a useful analysis on a rather tiny data set (six data values, in the case of the three-day series we analyze). In doing so, to augment the limited information in the data, we must assume a rather informative model for the turtles' nesting behavior. Fortunately, knowledge of the biological nesting process is trustworthy, and we maintain that this scientific knowledge should be heavily relied upon in the estimation process. Our Bayesian approach is ideal for marrying the information in the data with the knowledge of the nesting process.

The subject-matter knowledge we have incorporated into our priors is specific to turtle behavior off the coast of the Southeastern United States (and around the time our data set was gathered). Our method is generalizable: It can estimate loggerhead population sizes for analogous data sets collected at other locations or during other years. The parameters of our priors can be changed to reflect characteristics of the turtles in such locations and such years, and the estimation can be carried out analogously. Thus our approach can give ecologists trustworthy estimation of the abundance of this threatened species, as well as (more generally) providing insight into incorporating subject matter knowledge in a statistical analysis. 


\section{Acknowledgments}

We thank DuBose Griffin of the South Carolina Department of Natural Resources for providing the data set and insight about the turtles' nesting behavior.

\section{Appendix}

We now justify our expression for the full conditional for $\mu$ from Section 3.2. First, our assertion that $N_{o b s} /\left(N_{O O O}+N_{o b s}\right)$ carries the same information about $\mu$ as $\left(N_{O O O}, \mathbf{m}, \mathbf{y}\right)$ is argued as follows. First note that the relative sizes of the individual elements of $\mathbf{m}$ (and of $\mathbf{y}$ ) are unrelated to $\mu$ since they relate strictly to onshore behavior of the turtles rather than offshore behavior. In addition, $N_{o b s}=\sum_{i=1}^{3} m_{i}$ is also noninformative in isolation. However, consider the bivariate transformation from $\left(N_{O O O}, N_{o b s}\right)$ to $\left(N_{o b s} /\left(N_{O O O}+N_{o b s}\right), N_{O O O}+N_{o b s}\right)$. By itself, the statistic $N_{t o t}=N_{O O O}+N_{o b s}$ can be viewed as ancillary for $\mu$ since $\mu$ is a biological characteristic of individual turtles, independent of the overall size $N_{t o t}$ of the turtle population under study.

We thus wish to approximate the distribution of $N_{o b s} /\left(N_{O O O}+N_{o b s}\right)$. We argue that the probability of a random turtle being offshore all three days is approximately

$$
\frac{\mu-2}{\mu+1+q / p}
$$

the denominator being the expected length of the entire offshore-false crawl-nest cycle. (Intuitively, note that if a turtle stays offshore for $\mu$ days, it will fall in the $O O O$ category if the survey begins on one of the first $\mu-2$ of its offshore days.) Therefore, it is sensible to equate $\hat{\xi}=N_{o b s} /\left(N_{O O O}+N_{o b s}\right)$ to

$$
\xi=1-\frac{\mu-2}{\mu+1+q / p}=\frac{3+q / p}{\mu+1+q / p}
$$

Since $\hat{\xi}=N_{o b s} /\left(N_{O O O}+N_{o b s}\right)$ is a sample proportion, when the denominator is 
large we can assume that

$$
\hat{\xi} \dot{\sim} N\left(\xi, \frac{\frac{3+q / p}{\mu+1+q / p}\left(1-\frac{3+q / p}{\mu+1+q / p}\right)}{N_{O O O}+N_{o b s}}\right),
$$

By the delta method, where

$$
g(\xi)=\mu=\frac{1}{\xi}(3+q / p)-(1+q / p)
$$

we have that

$$
g(\hat{\xi})=\frac{\left(N_{O O O}+N_{o b s}\right)}{N_{o b s}}(3+q / p)-(1+q / p) \dot{\sim} N\left(g(\xi),\left[g^{\prime}(\xi)\right]^{2} V(\hat{\xi})\right)
$$

and $\left[g^{\prime}(\xi)\right]^{2}=(3+q / p)^{2} / \xi^{4}=(\mu+1+q / p)^{4} /(3+q / p)^{2}$. Therefore after estimating the $\xi=\frac{3+q / p}{\mu+1+q / p}$ in the variance expression in (4) with $\hat{\xi}=\frac{N_{\text {obs }}}{\left(N_{O O O}+N_{o b s}\right)}$, we have $\frac{\left(N_{O O O}+N_{o b s}\right)}{N_{o b s}}(3+q / p)-(1+q / p) \dot{\sim} N\left(\mu, \frac{(3+q / p)^{2}\left(N_{O O O}+N_{o b s}\right)^{4}}{N_{o b s}^{4}} \frac{\frac{N_{o b s}}{N_{O O O}+N_{o b s}} \frac{N_{O O O O}}{N_{O O O}+N_{o b s}}}{N_{O O O}+N_{o b s}}\right)$ and simplifying yields

$$
\frac{\left(N_{O O O}+N_{o b s}\right)}{N_{o b s}}(3+q / p)-(1+q / p) \dot{\sim} N\left(\mu, \frac{(3+q / p)^{2}\left(N_{O O O}+N_{o b s}\right) N_{O O O}}{N_{o b s}^{3}}\right) .
$$

Therefore

$$
\begin{aligned}
f\left(\mu \mid \mathbf{m}, N_{O O O}\right) & \propto f(g(\hat{\xi}) \mid \mu) f(\mu) \\
& \propto \exp \left\{-\frac{1}{2 V}(\mu-M)^{2}\right\} \exp \left\{-\frac{1}{2 \sigma_{\mu}^{2}}\left(\mu-\mu_{\mu}\right)^{2}\right\}
\end{aligned}
$$

where $M=(3+q / p)\left(N_{O O O}+N_{o b s}\right) / N_{o b s}-(1+q / p)$ and $V=\left[(3+q / p)^{2}\left(N_{O O O}+\right.\right.$ $\left.\left.N_{o b s}\right) N_{O O O}\right] / N_{o b s}^{3}$.

Standard calculations for Bayesian normal-normal models show this reduces to a distribution that is

$$
N\left(\frac{\frac{\mu_{\mu}}{\sigma_{\mu}^{2}}+\frac{M}{V}}{\frac{1}{\sigma_{\mu}^{2}}+\frac{1}{V}}, \frac{\sigma_{\mu}^{2} V}{\sigma_{\mu}^{2}+V}\right) .
$$




\section{References}

[1] Agresti, A. (2002). Categorical Data Analysis. Hoboken, NJ: John Wiley \& Sons.

[2] Brooks, S. P., Catchpole, E. A., and Morgan B. J. T. (2000). "Bayesian Animal Survival Estimation," Statistical Science, 15, 357-376.

[3] Cormack, R. M. (1989). "Log-linear Models for Capture-recapture," Biometrics, $45,395-413$.

[4] Crouse, D. T. (1984). "Loggerhead Sea Turtle Nesting in North Carolina: Applications of an Aerial Survey." Biological Conservation, 29, 143-155.

[5] Cummings, N. E. (2011). "Bayesian Mark-recapture Analysis," ISBA Bulletin, $18,6-8$.

[6] Dodd, C. K. (1988). "Synopsis of the Biological Data on the Loggerhead Sea Turtle Caretta caretta (Linnaeus 1758)." Biological Report 88 (14) (FAO Synopsis NMFS-149, United States Fish and Wildlife Service): 183.

[7] Dupuis, J. A. (1995). "Bayesian Estimation of Movement and Survival Probabilities from Capture-recapture Data," Biometrika, 82, 761-772.

[8] Evans, M. A., Bonett, D. G., and McDonald, L. L. (1994). "A General Theory for Modeling Capture-recapture Data from a Closed Population," Biometrics, 50, 396-405.

[9] Fienberg, S. E. (1972). "The Multiple-recapture Census for Closed Populations and Incomplete $2^{k}$ Contingency Tables," Biometrika, 59, 591-603.

[10] Frazer, N. B. and Richardson, J. I. (1985). "Annual Variation in Clutch Size and Frequency for Loggerhead Turtles, Caretta caretta, nesting at Little Cumberland Island, Georgia, USA," Herpetologica, 41, 246-251. 
[11] Gallagher, R.M., Hollinger, M. L., Ingle, R. M., and Futch, C. R. (1972). "Marine Turtle Nesting on Hutchinson Island, Florida, in 1971," Florida Department of Natural Resources Scientific Report, 37.

[12] Gazey, W. J. and Staley, M. J. (1986). "Population Estimation from Markrecapture Experiments using a Sequential Bayes Algorithm," Ecology, 67, 941951.

[13] George, E. I. and Robert, C. P. (1992). "Capture-recapture Estimation via Gibbs Sampling," Biometrika, 79, 677-683.

[14] Hopkins-Murphy, S. R., Murphy, T. M., Hope, C. P., Coker, J. W., Hoyle, M. E. (2001). "Population Trends and Nesting Distribution of the Loggerhead Turtle (Caretta caretta) in South Carolina 1980-1997: Final Report to the U.S. Fish and Wildfile Service," South Carolina Department of Natural Resources Report.

[15] Limpus, C. J. and Reed, P. C. (1985). "The loggerhead turtle, Caretta caretta, in Queensland: Observations on Internesting Behaviour," Australian Wildlife Research, 12, 535-540.

[16] Margaritoulis, D. (1985). "Preliminary Observations on the Breeding Behaviour and Ecology of Caretta caretta in Zakynthos, Greece," Biologia Gallo-hellenica, 10, 323-332.

[17] McClave, J. T. and Sincich, T. (2006). Statistics, 10th edition. Upper Saddle River, NJ: Pearson/Prentice Hall.

[18] Murphy, S. and Griffin, D. (2005). "Loggerhead Turtle: Caretta caretta," South Carolina Department of Natural Resources Report. Available at http://www.dnr.sc.gov/cwcs/pdf/Loggerheadturtle.pdf (Accessed May 2012). 
[19] NOAA Office of Protected Resources (2011). "Endangered and Threatened Species under NMFS' Jurisdiction," National Oceanic and Atmospheric Administration National Marine Fisheries Services Report. Available at http://www.nmfs.noaa.gov/pr/pdfs/species/esa_table.pdf (Accessed May 2012).

[20] Otis, D. L., Burnham, K. P., White, G. C., and Anderson, D. R. (1978). "Statistical Inference from Capture Data on Closed Animal Populations." Wildlife Monographs, 62, 1135.

[21] Pledger, S., Pollock, K.H, and Norris, J.L. (2003). "Open Capture-Recapture Models with Heterogeneity: I. Cormack-Jolly-Sieber Model." Biometrics, 59, 786-794.

[22] Rasch, G. (1961). "On General Laws and the Meaning of Measurement in Psychology," In: Proceedings of 4th Berkeley Symposium on Mathematical Statistics and Probability, (Ed: J. Neyman). Berkeley, CA: University of California Press, 4, pp. 321-333.

[23] Raymond P. W. (1984). "The Effects of Beach Restoration on Marine Turtles Nesting in South Brevard County, Florida," M.S. Thesis, University of Central Florida.

[24] Richardson, J. I. and Richardson, T. H. (1982). "An Experimental Population Model for the Loggerhead Sea Turtle (Caretta caretta)," In: Biology and Conservation of Sea Turtles (ed: K. Bjorndal), 165-176. Washington: Smithsonian Institution Press.

[25] Ross, S. M. (1996). Stochastic Processes. New York: John Wiley \& Sons.

[26] Stoneburner, D. L. (1981). "Summary of the Loggerhead Sea Turtle Research Project Conducted at Canaveral National Seashore, Cumberland Island National 
Seashore, Cape Lookout National Seashore: A Final Report 1980." National Park Service Res./Resource Management Report, 39.

[27] Stoneburner, D. L. and Richardson, J. I. (1981). "Observations on the Role of Temperature in Loggerhead Nest Site Selection," Copeia, 1981, 238-241.

[28] Talbert, O. R., Jr., Stancyk, S. E., Dean, J. M., and Will, J. M. (1980). "Nesting Activity of the Loggerhead Turtle (Caretta caretta) in South Carolina. I: a rookery in transition." Copeia, 1980, 709-719. 


\begin{tabular}{cc|ccc} 
& & \multicolumn{4}{|c}{ Day 3 } \\
\hline Day 1 & Day 2 & $\mathrm{O}$ & $\mathrm{F}$ & $\mathrm{N}$ \\
\hline $\mathrm{O}$ & $\mathrm{O}$ & $\frac{\mu \omega^{2}}{\mu+1+q / p}$ & $\frac{\mu \omega q(1-\omega)}{\mu+1+q / p}$ & $\frac{\mu \omega p(1-\omega)}{\mu+1+q / p}$ \\
\hline $\mathrm{O}$ & $\mathrm{F}$ & 0 & $\frac{\mu q^{2}(1-\omega)}{\mu+1+q / p}$ & $\frac{\mu q(1-\omega) p}{\mu+1+q / p}$ \\
\hline $\mathrm{O}$ & $\mathrm{N}$ & $\frac{\mu p(1-\omega)}{\mu+1+q / p}$ & 0 & 0 \\
\hline $\mathrm{F}$ & $\mathrm{O}$ & 0 & 0 & 0 \\
\hline $\mathrm{F}$ & $\mathrm{F}$ & 0 & $\frac{q^{3} / p}{\mu+1+q / p}$ & $\frac{q^{2}}{\mu+1+q / p}$ \\
\hline $\mathrm{F}$ & $\mathrm{N}$ & $\frac{q}{\mu+1+q / p}$ & 0 & 0 \\
\hline $\mathrm{N}$ & $\mathrm{O}$ & $\frac{1}{\mu+1+q / p}$ & 0 & 0 \\
\hline $\mathrm{N}$ & $\mathrm{F}$ & 0 & 0 & 0 \\
\hline $\mathrm{N}$ & $\mathrm{N}$ & 0 & 0 & 0 \\
\hline
\end{tabular}

Table 1: Table of joint probabilities for successive three-day outcomes $A B C$, where $A$ is the turtle's state on Day $1, B$ is the turtle's state on Day 2, and $C$ is the turtle's state on Day 3. 


\begin{tabular}{|c|c|c|c|}
\hline & $\mu_{\mu}=12$ & $\mu_{\mu}=13$ & $\mu_{\mu}=14$ \\
\hline \multirow[t]{6}{*}{$\sigma_{\mu}=0.5$} & 0.68 & 0.90 & 0.85 \\
\hline & $(0.033)$ & $(0.021)$ & $(0.025)$ \\
\hline & 149.44 & 155.86 & 161.48 \\
\hline & $(0.32)$ & $(0.33)$ & $(0.30)$ \\
\hline & -58.98 & -7.62 & 32.33 \\
\hline & $(2.97)$ & $(3.28)$ & $(2.96)$ \\
\hline \multirow[t]{6}{*}{$\sigma_{\mu}=0.75$} & 0.805 & 0.955 & 0.91 \\
\hline & $(0.028)$ & $(0.015)$ & $(0.020)$ \\
\hline & 163.60 & 169.17 & 173.19 \\
\hline & $(0.35)$ & $(0.35)$ & $(0.39)$ \\
\hline & -47.29 & 1.38 & 28.55 \\
\hline & $(2.80)$ & $(2.99)$ & $(3.37)$ \\
\hline \multirow[t]{6}{*}{$\sigma_{\mu}=1$} & 0.895 & 0.985 & 0.94 \\
\hline & $(0.022)$ & $(0.009)$ & $(0.017)$ \\
\hline & 177.27 & 181.66 & 185.02 \\
\hline & $(0.35)$ & $(0.35)$ & $(0.38)$ \\
\hline & -39.91 & -1.50 & 25.55 \\
\hline & $(2.56)$ & $(2.58)$ & $(2.86)$ \\
\hline
\end{tabular}

Table 2: Effect of specification of prior on $\mu$. Quality measures [Top of cell: Coverage probability. Middle of Cell: Average Width. Bottom of Cell: Bias.] for 90\% Credible Intervals and Point Estimates, averaged across 200 simulated data sets per setting. (Monte Carlo standard errors for the measures are given in parentheses.) 


\begin{tabular}{|c|c|c|c|}
\hline & mean $=700$ & mean $=755$ & mean $=800$ \\
\hline \multirow[t]{6}{*}{$\operatorname{size}=200$} & 0.815 & 0.955 & 0.935 \\
\hline & $(0.028)$ & $(0.015)$ & $(0.017)$ \\
\hline & 132.43 & 138.33 & 142.97 \\
\hline & $(0.19)$ & $(0.21)$ & $(0.18)$ \\
\hline & -40.34 & -5.89 & 19.48 \\
\hline & $(2.22)$ & $(2.44)$ & $(2.31)$ \\
\hline \multirow[t]{6}{*}{$\operatorname{size}=80$} & 0.89 & 0.955 & 0.96 \\
\hline & $(0.022)$ & $(0.015)$ & $(0.014)$ \\
\hline & 163.19 & 169.17 & 172.71 \\
\hline & $(0.32)$ & $(0.35)$ & $(0.35)$ \\
\hline & -35.10 & 1.38 & 14.08 \\
\hline & $(2.66)$ & $(2.99)$ & $(3.00)$ \\
\hline \multirow[t]{6}{*}{ size $=40$} & 0.915 & 0.94 & 0.95 \\
\hline & $(0.020)$ & $(0.017)$ & $(0.015)$ \\
\hline & 184.15 & 187.53 & 189.63 \\
\hline & $(0.44)$ & $(0.47)$ & $(0.50)$ \\
\hline & -23.91 & -9.54 & -2.82 \\
\hline & (3.13) & (3.38) & $(3.46)$ \\
\hline
\end{tabular}

Table 3: Effect of specification of prior on $N_{O O O}$. Quality measures [Top of cell: Coverage probability. Middle of Cell: Average Width. Bottom of Cell: Bias.] for 90\% Credible Intervals and Point Estimates, averaged across 200 simulated data sets per setting. (Monte Carlo standard errors for the measures are given in parentheses.) 


\begin{tabular}{|c|c|c|c|}
\hline & \multicolumn{3}{|c|}{ Measure Of Inferential Quality } \\
\hline $\begin{array}{l}\text { Distribution } \\
\text { for } p\end{array}$ & $\begin{array}{c}\text { Empirical Coverage } \\
\text { Probability }\end{array}$ & $\begin{array}{l}\text { Average } \\
\text { Width }\end{array}$ & $\begin{array}{c}\text { Bias (of } \\
\text { posterior median) }\end{array}$ \\
\hline 0.6 (constant) & $\begin{array}{c}0.955 \\
(0.015)\end{array}$ & $\begin{array}{l}169.17 \\
(0.35)\end{array}$ & $\begin{array}{c}1.38 \\
(2.99)\end{array}$ \\
\hline $\operatorname{beta}(6,4)$ & $\begin{array}{c}0.965 \\
(0.013)\end{array}$ & $\begin{array}{l}168.37 \\
(0.33)\end{array}$ & $\begin{array}{l}-10.33 \\
(2.83)\end{array}$ \\
\hline $\operatorname{beta}(9,6)$ & $\begin{array}{c}0.975 \\
(0.011)\end{array}$ & $\begin{array}{l}168.24 \\
(0.32)\end{array}$ & $\begin{array}{l}-9.09 \\
(2.64)\end{array}$ \\
\hline $\operatorname{beta}(12,8)$ & $\begin{array}{c}0.92 \\
(0.018)\end{array}$ & $\begin{array}{l}168.23 \\
(0.39)\end{array}$ & $\begin{array}{l}-2.92 \\
(3.41)\end{array}$ \\
\hline
\end{tabular}

Table 4: Robustness to non-constant nesting probability. Quality measures for 90\% Credible Intervals and Point Estimates, averaged across 200 simulated data sets per setting. (Monte Carlo standard errors for the measures are given in parentheses.)

\begin{tabular}{lccc} 
& \multicolumn{3}{c}{ Day } \\
\hline \hline & 1 & 2 & 3 \\
\hline Nests & 77 & 76 & 71 \\
\hline False Crawls & 58 & 87 & 72 \\
\hline
\end{tabular}

Table 5: Table of counts of nests and false crawls: June 16-18, 2000. 

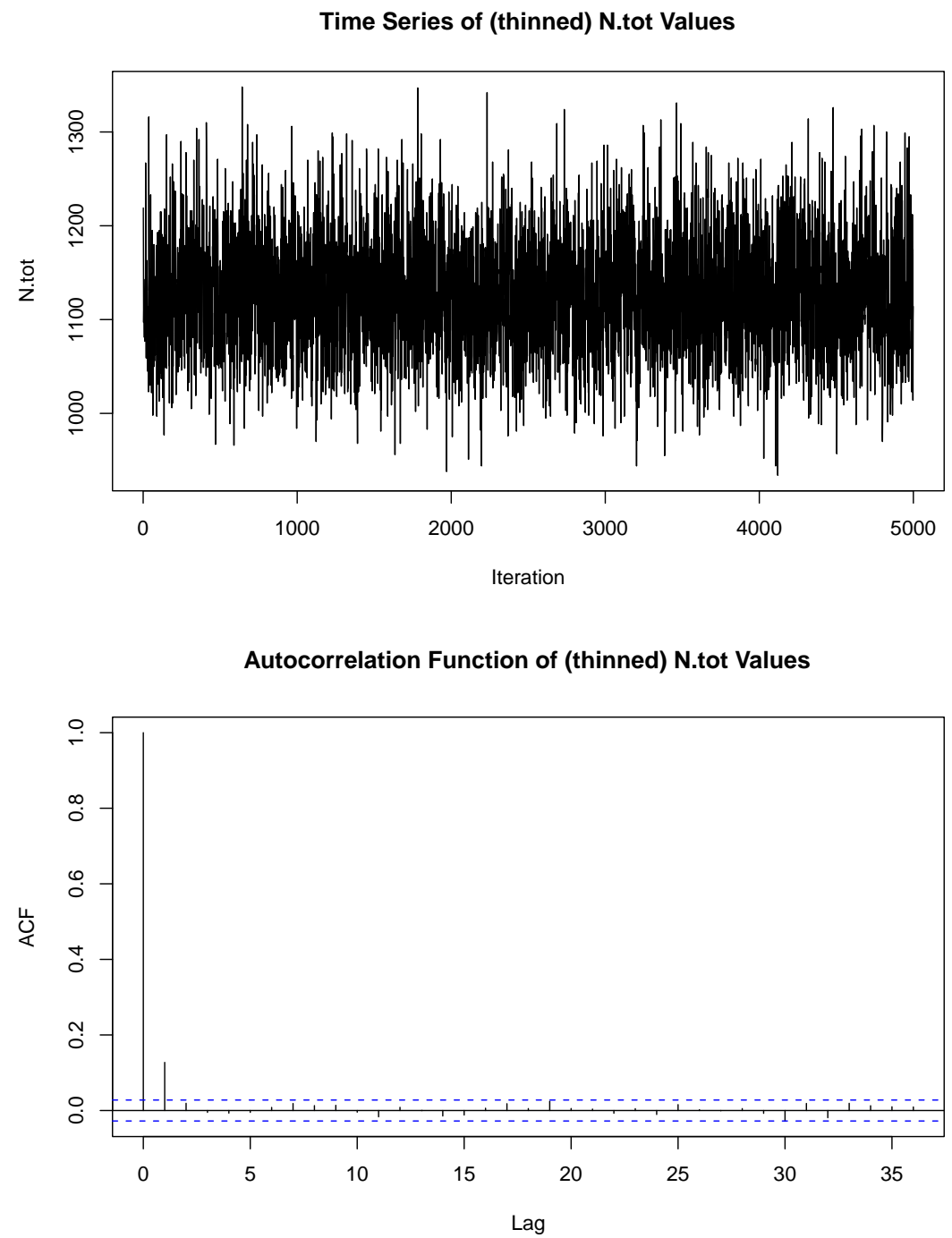

Figure 1: Time series plot (top) and autocorrelation function (bottom) of (thinned) posterior sample of $N_{\text {tot }}$ values: June 16-18, 2000. 
Draws from Posterior of N.tot (thinned)

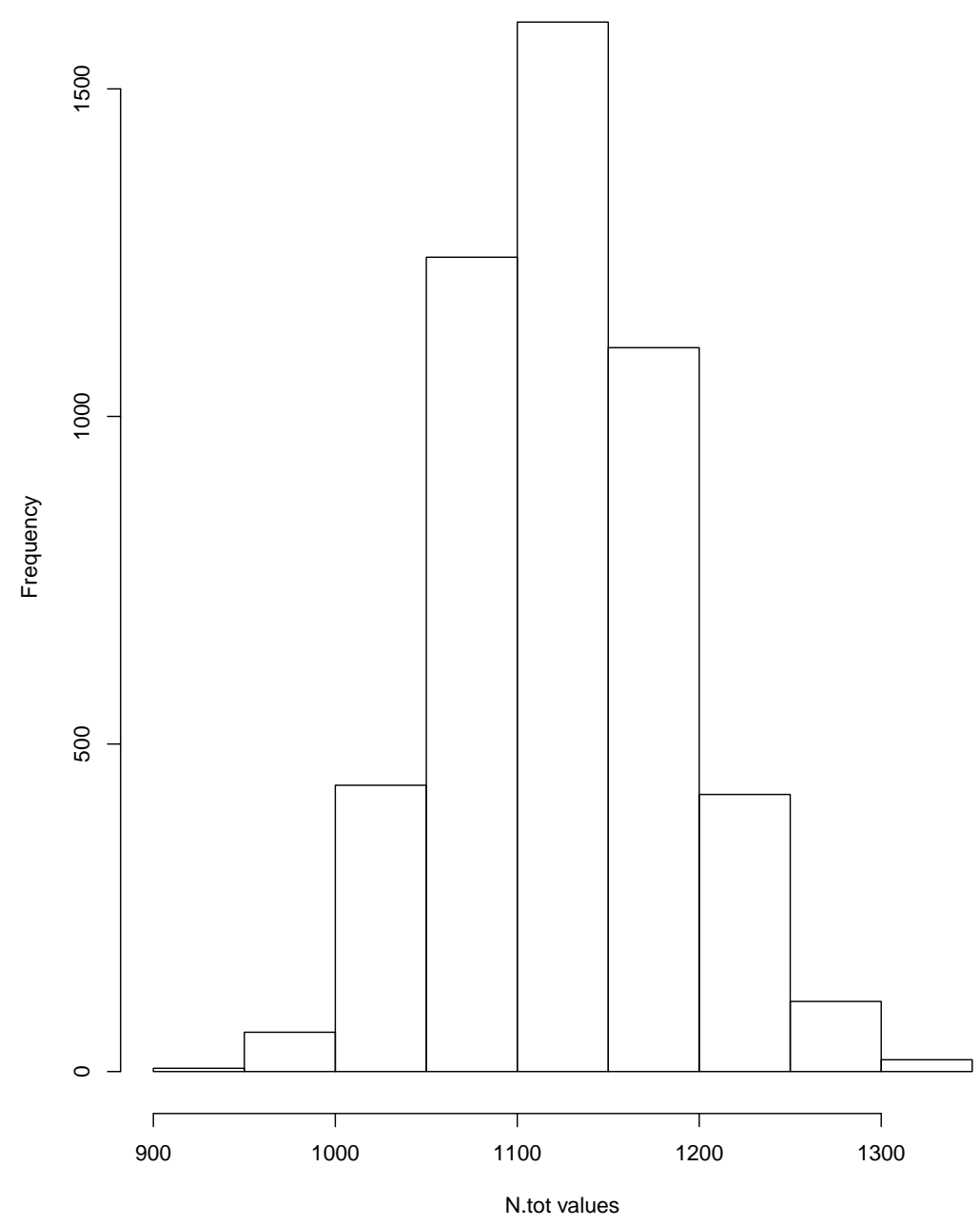

Figure 2: Histogram of (thinned) posterior sample of $N_{\text {tot }}$ values: June 16-18, 2000. 